OPEN ACCESS

Edited by:

Tetsuo Kida

National Institute for Physiological

Sciences, Japan

Reviewed by:

Annette Horstmann,

Max Planck Institute for Human

Cognitive and Brain Sciences,

Germany

Jennifer Silvers,

University of California, Los Angeles,

USA

*Correspondence:

Robert Hester

hesterr@unimelb.edu.au

Received: 17 May 2016 Accepted: 10 August 2016 Published: 26 August 2016

Citation:

Scalzo F, O'Connor DA, Orr C, Murphy $K$ and Hester R (2016)

Attention Diversion Improves

Response Inhibition of Immediate

Reward, But Only When it Is

Beneficial: An fMRI Study.

Front. Hum. Neurosci. 10:429.

doi: 10.3389/fnhum.2016.00429

\section{Attention Diversion Improves Response Inhibition of Immediate Reward, But Only When it Is Beneficial: An fMRI Study}

\author{
Franco Scalzo ${ }^{1}$, David A. O'Connor ${ }^{1,2}$, Catherine Orr ${ }^{1,3}$, Kevin Murphy ${ }^{4}$ and \\ Robert Hester ${ }^{1 *}$
}

${ }^{1}$ Melbourne School of Psychological Sciences, University of Melbourne, Melbourne, VIC, Australia, ${ }^{2}$ Cognitive Neuroscience Centre, Reward and Decision-Making Group, Centre National pour la Recherche Scientifique, Lyon, France, ${ }^{3}$ Departments of Psychiatry and Psychology, University of Vermont, Burlington, VT, USA, ${ }^{4}$ Cardiff University Brain Research Imaging Centre, School of Psychology, Cardiff University, Cardiff, UK

Deficits of self-control are associated with a number of mental state disorders. The ability to direct attention away from an alluring stimulus appears to aid inhibition of an impulsive response. However, further functional imaging research is required to assess the impact of shifts in attention on self-regulating processes. We varied the level of attentional disengagement in an functional magnetic resonance imaging (fMRI)based Go/No-go task to probe whether diversion of attention away from alluring stimuli facilitates response inhibition. We used the attention-grabbing characteristic of faces to exogenously direct attention away from stimuli and investigated the relative importance of attention and response inhibition mechanisms under different delayed reward scenarios [i.e., where forgoing an immediate reward (\$1) led to a higher (\$10) or no payoff in the future]. We found that diverting attention improved response inhibition performance, but only when resistance to an alluring stimulus led to delayed reward. Region of interest analyses indicated significant increased activity in posterior right inferior frontal gyrus during successful No-go trials for delayed reward trials compared to no delayed reward trials, and significant reduction in activity in the superior temporal gyri and left caudate in contexts of high attentional diversion. Our findings imply that strategies that increase the perceived benefits of response inhibition might assist individuals in abstaining from problematic impulsive behaviors.

Keywords: attention, response inhibition, reward, Go/No-go task, inferior frontal gyrus, superior temporal gyrus, fMRI

\section{INTRODUCTION}

An important facet of human cognition is an individual's capacity to exercise self-control. The ability to refrain from inappropriate behaviors facilitates effective interaction with society, care for one's self, and pursuit of longer-term goals at the expense of immediate gratification (Mischel et al., 1988; Baumeister and Heatherton, 1996). Deficits in self-control are associated with a number of health issues including substance abuse (Fillmore and Rush, 2002; Monterosso et al., 2005; Goudriaan et al., 2006) and mental state disorders (Penadés et al., 2007; Boonstra et al., 2010). Selfcontrol has been characterized as a balance between long-term goals and immediate temptations 
(Ochsner and Gross, 2005; Li and Sinha, 2008; Heatherton and Wagner, 2011). There is also evidence that visual attention is important for controlling impulsive responses to immediately available rewards (Mischel and Ebbesen, 1970; Mischel et al., 1972; Casey et al., 2011). For instance, resistance to a tempting stimulus can be prolonged by focussing on less tempting features of the target (Mischel and Baker, 1975), or deploying attention away from the target (Mischel and Ebbesen, 1970; Mischel et al., 1972). Conversely, by controlling visual fixations toward appetitive items, attention has been shown to bias choices for fixated items (Armel et al., 2008; Lim et al., 2011). However, despite the wealth of research in this field, the question of whether inhibition of motor responses over immediately available rewards can be improved by diverting attention away from them has not yet been explored. Moreover the neural correlates of such a phenomenon remain unexamined.

Response inhibition has been functionally well-defined in the prefrontal cortex (PFC), particularly the right inferior frontal gyrus (rIFG; Aron, 2011; Tabibnia et al., 2011), although others suggest a more general role for the rIFG (Hampshire and Sharp, 2015). On the other hand, the dorsal striatum is implicated in reward-related motivational and learning processes for goaldirected behavior (Balleine et al., 2007; Padmala and Pessoa, 2010; O'Connor et al., 2012). It is theorized that self-regulatory failure is more likely when a striatal response prevails over a PFC response (Heatherton and Wagner, 2011). For instance, exposure to highly alluring cues (e.g., offered your favorite chocolate) may overwhelm PFC response, or PFC function may be impaired (e.g., due to negative mood; Peters and Büchel, 2011). Is it also possible that, by reducing the saliency of stimuli and related striatal response, the probability of self-regulatory failure can be decreased? Hypoactivity in reward-related neural areas has been accompanied by hypoactivity in neural regions associated with attention during successful response inhibition or craving resistance (Volkow et al., 2010; O'Connor et al., 2012). These regions include the right superior temporal gyrus (rSTG), which has been associated with shifting attention in tasks that involve relative value coding (Hampton et al., 2008; Hare et al., 2010; Lim et al., 2013).

We have previously argued that the ability to direct attention away from an alluring stimulus may be an endogenous mechanism that assists inhibitive control over that stimulus when there is a reason to do so (O'Connor et al., 2012). If an individual can direct attention away from a cue, then the prepotency of an alluring stimulus is reduced and inhibition may be easier, requiring less involvement of rIFG than might otherwise be the case (O'Connor et al., 2012). During employment of cognitive control strategies designed to resist cue-induced craving, hypoactivation of visual processing areas was accompanied by either no significant change to rIFG activation (Brody et al., 2007) or rIFG hyperactivity (Volkow et al., 2010). The results may be further evidence that for improved understanding of PFC-related resistance to reward, attentional mechanisms should be taken into account.

In this functional magnetic resonance imaging (fMRI) study, we modified a Go/No-go task to investigate whether response inhibition can be enhanced by exogenously diverting attention away from immediately rewarding target stimuli. In addition to standard Go trials requiring a button press response, we also introduced Go-Money trials which, when responded to with sufficient speed, provided immediate feedback for a small monetary reward. A subset of Go-Money trials was visually modified to indicate that participants had to inhibit their standard rapid rewarding responses (No-go trials). A response to No-go trials was considered a failure of response inhibition. Crucially, No-go trials could be accompanied by either a high or low means of diverting attention. This manipulation allowed us to investigate whether diverting attention from an immediately rewarding stimulus improves response inhibition. Processing faces appears to be automatic and mandatory (Farah, 1996; Vuilleumier and Schwartz, 2001; Lavie et al., 2003) and were therefore chosen to exogenously divert attention away from No-go trials. To contrast these high-level attention diversion No-go trials, scrambled faces accompanied the remaining Nogo trials. Because scrambled faces do not possess the same attention-capturing characteristics, these served as low-level attention diversion No-go trials. Finally, No-go trials were further manipulated to address another important question. Specifically, if diverting attention away from immediately rewarding stimuli is actually effective in improving response inhibition, is this enhancement only effective if there is a larger potential reward available in the future? To explore this possibility, we compared performance for No-go trials where successful response inhibition would contribute to a larger reward but where no immediate feedback is given, against performance on No-go trials where no such delayed reward contingencies were available. In this way, the paradigm was designed to include a model of real-life circumstances in which abstinence is required over an immediate and tangible reward, in order to obtain a larger, less tangible, future benefit. In parallel with a hypothesized behavioral improvement in response inhibition over immediately rewarding stimuli as a result of attention diversion, we hypothesized modulation of brain regions relevant to cognitive control (PFC), reward (striatum), and attention (STG).

\section{MATERIALS AND METHODS}

\section{Participants}

Twenty-six volunteers participated in this study, recruited from the community through advertising. Five participants were excluded from data analyses due to either non-completion of scan (one), excessive head movement during structural scans (three), or identification of anomalous anatomical features (one). Twenty-one healthy volunteers (13 females and eight males; $M_{\text {age }}=24.7$ years, $S D=4.9$, range $=17.7-33.5$ ) were included in the data analyses. All were right-handed, as determined by the Edinburgh Handedness Inventory (Oldfield, 1971), and reported no current or past history of neurological or psychiatric disorders or psychotropic medication use. All participants, and a parent or guardian for those aged less than 18 years, provided written informed consent and were screened for physical or medical conditions affecting eligibility for magnetic resonance 
imaging (MRI) scanning. The University of Melbourne Human Ethics Committee approved the study protocol. Participants were compensated $\$ 20 / \mathrm{h}$ for their participation, plus $5 \%$ of the amount earned during the Go/No-go task. The average performance bonus was $\$ 34$.

\section{Go/No-go Task Key Condition Manipulations}

The modified Go/No-go task consisted of two key condition manipulations (Figure 1). First, we examined the notion that diversion of attention away from an alluring stimulus might facilitate response inhibition by utilizing the assumed attentiongrabbing attributes of faces. Second, we utilized a manipulation of delayed reward to test whether the proposed shift in attention away from immediately rewarding targets to aid response inhibition is exclusive to situations in which successful control of impulses yields a future benefit. The task manipulated future reward insofar as for half of the No-go events, successful response inhibition produced no delayed reward. In addition to these key manipulations, we also developed alluring reward-response associations for targets by providing small immediate monetary rewards and feedback for successful Go-Money and unsuccessful No-go trials.

For each trial, participants were presented with an image of a building. Two characteristics of the building stimulus determined whether participants should respond (Go trials), respond rapidly for a reward (Go-Money trials) or withhold their response (Nogo trials). These characteristics were the building type (house, church, or castle) and whether lights were on or off in the windows of the building. Go trials, represented by houses, always required a response from participants. Neither reward nor performance feedback was provided for Go trials, so the target was not alluring. The second most frequent trials to appear, GoMoney trials, were represented by churches and castles. Upon presentation of Go-Money trials, participants were required to respond rapidly. A rapid response ensured immediate monetary reward feedback of $\$ 1$ and meant that Go-Money trials were alluring. Finally, the least frequent type of trial, No-go trials, were also represented by churches and castles. This conflation with GoMoney trials was intentional as it meant that associations with immediate reward feedback were held constant and, therefore, No-go trials remained alluring. Indeed, failure to inhibit Nogo trials still led to an immediate reward outcome of $\$ 1$. The only characteristic that differentiated Go-Money trials from Nogo trials was whether the windows of the building showed lights to be "on" or "off." Therefore, depending on instructions at the beginning of the experimental block, participants could respond rapidly to presentation of a church with lights "on" in one trial and then withhold response to presentation of a church with lights "off" in another trial. This conflation and nuanced difference also ensured that participants had to attend fully to each stimulus in order to attain the appropriate behavior.

As previously outlined, experimental conditions varied on two factors: (a) high and low attention diversion; and (b) large delayed reward and no delayed reward for successful response

\section{A Go/No-go Stimuli: Examples}

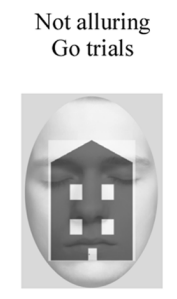

House: No rewards
Alluring

Go-Money trials

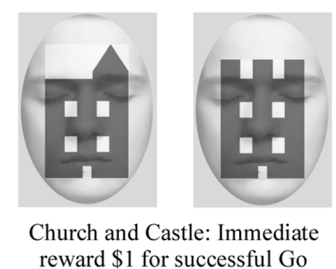

B Example of stimuli with lights on or off in windows

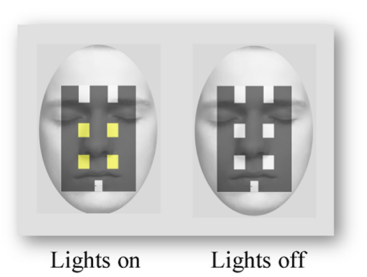

\section{Experimental manipulations}

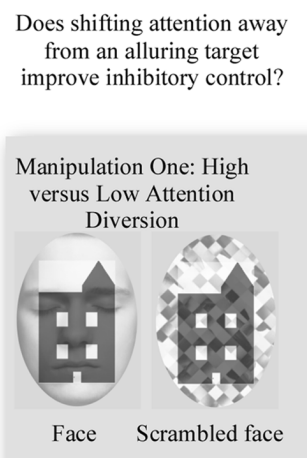

Is diversion from an alluring stimulus only helpful when the consequence of the inhibition ultimately leads to something?

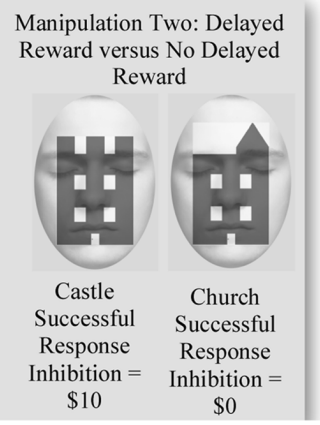

FIGURE 1 | Examples of the visual stimuli used for the Go/No-go task and experimental manipulations. (A) Examples of target stimuli. No reward was associated with successful response to house (Go) trials, and an immediate one dollar reward was provided for successful response to church or castle (Go-Money) trials. (B) Example of use of lights for target stimuli. No-go trials were defined by whether a church or castle had lights on/off in their windows. (C) Examples illustrate manipulation of attention diversion by using a face and scrambled face in the background as high and low attention diversion, respectively. Successful response inhibition of a castle resulted in a ten-dollar reward, whereas successful response inhibition of a church yielded no reward.

inhibition. For (a) high and low attention diversion, stimuli were presented with a background face or scrambled face, respectively. Although we were only interested in the effect of attention diversion on No-go performance, face/scrambled face backgrounds were applied to all trials to ensure that participants could not identify No-go trials simply by the presence of a face or scrambled face. To minimize emotional engagement and possible related interference in amygdalae, a passive face with eyes closed was used. Faces were fitted to an oval frame and 
placed behind relevant stimuli. For (b) large delayed reward and no delayed reward for successful response inhibition, the concept of a "delayed" or future reward was simulated through the absence of any immediate feedback for successful response inhibition. In this way, the requirement to inhibit a response was not tangibly alluring, as it provided no concrete feedback about reward outcomes. Moreover, both delayed and no delayed reward conditions had the same potent immediate reward association, so that failure to withhold an impulse yielded the previously learned immediate and tangible reward. Instead, participants were simply instructed that for each successful response inhibition of a Nogo trial represented by a castle, they would earn a $\$ 10$ reward, whereas each successful response inhibition of a No-go trial represented by a church would earn no such reward. Therefore, the condition of delayed reward for successful inhibition was modeled to be closest to a real-life situation, including the contingency that failure to abstain yielded some immediate, small reward. That is, abstinence was required over an immediate and tangible reward to obtain a larger, but less tangible, future benefit.

\section{Task Design}

Prior to entering the MRI scanner, participants were given detailed instructions and were fully practiced on the task and its contingencies until they had a good understanding of it. The Go/No-go task consisted of eight blocks of trials with 180 trials per block, and used a ratio of 6:2:1 Go:Go-Money:Nogo responses (i.e., 120 Go, 40 Go-Money, and 20 No-go trials per block). In four of the eight blocks, Go-Money trials were defined as churches and castles with their lights "on," and Nogo trials were churches and castles with lights "off." In the other four blocks, Go-Money trials were churches/castles with lights "off," and No-go trials were churches/castles with lights "on." An event-related design permitted presentation of different trials in arbitrary sequences, thus reducing potential for habituation or anticipation (Rosen et al., 1998), and facilitated averaging across specific events. Background attention diversion and target stimuli were counterbalanced across the present experiment such that there were four sequences in total. No-go trials were pseudorandomly interspersed throughout the Go and Go-Money trials. The stimulus was presented for $750 \mathrm{~ms}$, followed by a $1000 \mathrm{~ms}$ interstimulus interval, and then a $500 \mathrm{~ms}$ fixation cross. For Go-Money and No-go trials, the fixation cross was preceded by a feedback screen for $750 \mathrm{~ms}$. In terms of feedback, a tick $(\sqrt{ })$ or cross $(\mathrm{X})$ was used to signify whether a response was correct or incorrect, respectively. Successful Go-Money trials, necessitated that response be within a time window of 100-400 ms. A response faster than $100 \mathrm{~ms}$ indicated high likelihood that the response preceded visual processing of the target (Macknik and Livingstone, 1998), and the $400 \mathrm{~ms}$ threshold was set to facilitate a spontaneous response. For Go-Money trials, participants received immediate feedback comprising a tick and one dollar reward for successful trials $(\sqrt{ } \$ 1.00)$ or that their response either took longer than $400 \mathrm{~ms}$ (Slow $\mathrm{X}$ $\$ 0.00$ ), or was faster than $100 \mathrm{~ms}$ (Fast X \$0.00). Successful withholding of a response to the castle yielded a tick and a 10-dollar reward although no feedback on amount earned for delayed reward was provided until the end of session. Failed inhibition of No-go trials resulted in immediate reward of one dollar ("X \$1.00"). There was neither feedback nor reward for House trials. An example of a typical sequence is shown in Figure 2. This portion of the task was approximately $60 \mathrm{~min}$ in duration.

To mitigate possible "reversal learning" effects from the light on/off manipulation between blocks of Go/No-go trials, each block was preceded by a "Go task" which required participants to respond to all (Go-Money and Go) targets, within 100$400 \mathrm{~ms}$. Each inter-block Go task was approximately $1 \mathrm{~min}$ in duration, and contained all stimuli in random order, with each target presented for $750 \mathrm{~ms}$, followed by a feedback screen for $750 \mathrm{~ms}$ for Go-Money trials, and then a fixation cross for $500 \mathrm{~ms}$. For Go-Money trials, participants received immediate feedback comprising a tick and one dollar reward for successful trials $(\sqrt{ } \$ 1.00)$ or that their response either took longer than $400 \mathrm{~ms}$ (Slow X \$0.00), or was faster than $100 \mathrm{~ms}$ (Fast $\mathrm{X} \$ 0.00)$. There was neither feedback nor reward for House trials.

\section{Apparatus}

Visual stimuli were presented using E-Prime software (version 2.0, Psychology Software Tools, Pittsburgh, PA, USA) on a laptop PC (Intel $2 \mathrm{Ghz}, 256 \mathrm{mb}$ Nvidia Video Card) that was interfaced with the magnetic resonance scanner during fMRI data acquisition. Stimuli were projected onto a screen located near the feet of the participant, who viewed the screen via a mirror attached to a 32 channel head coil. Behavioral responses were recorded using a scanner-compatible two-button box (FiberOptic response pad, Current Designs, Philadelphia, PA, USA). fMRI data were acquired at Swinburne University (Hawthorn, Australia) using a Siemens Tim Trio 3T MRI scanner (Erlangan, Germany).

\section{Functional Magnetic Resonance Imaging Data Acquisition}

Functional magnetic resonance imaging data for the Go/No-go tasks were acquired using 180 (gradient) echo-planar imaging (EPI) sequences which provided T2* (time constant for loss of signal in sequence). Weighted BOLD activity measurements were obtained for each functional run with the following parameters: repetition time $(\mathrm{TR})=2 \mathrm{~s}$; echo time $(\mathrm{TE})=36 \mathrm{~ms}$; flip angle $(\mathrm{FA})=90^{\circ} ; 192 \mathrm{~mm}$ field of view; and 38 contiguous slices of $4 \mathrm{~mm}$ slice thickness. An oblique $30^{\circ}$ orientation to the anterior-posterior commissure line was employed in data acquisition. The first two volumes of each run were discarded prior to data analysis to account for transients in the magnetic field of scanner. Eight functional runs were collected for each participant. At the completion of functional neuroimaging, high-resolution structural images were acquired using an MPRAGE T1-weighted sequence $[\mathrm{TR}=1900 \mathrm{~ms}$; $\mathrm{TE}=2.3 \mathrm{~ms}, \mathrm{FA}=90$, slice thickness $=0.90 \mathrm{~mm}$; inplane resolution $=1 \mathrm{~mm} \times 1 \mathrm{~mm}]$. During data processing, functional data were overlaid on the structural image for each participant, so that activations could be accurately localized with anatomy. 
Example: Do not respond if "lights off" for church or castle, otherwise respond

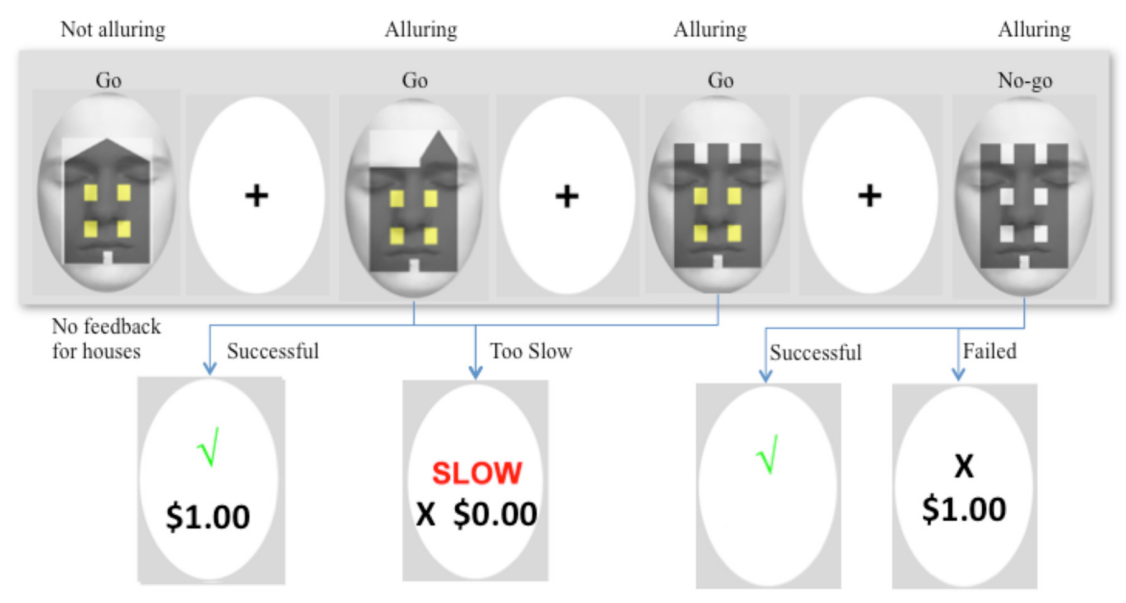

FIGURE 2 | Example of a typical sequence of the Go/No-go task. In this example, participants were instructed to withhold their response when a "castle" and "church" had lights "off" in their windows. The first target was a house, which required a "Go" response at all times. No reward or performance feedback was provided for the house and so the target was not alluring. The following two figures were a church and castle with lights "on," and both required a "Go" response. Each stimulus was presented for 750 ms, followed by a 1000 ms interstimulus interval, and then a 500 ms fixation cross. For Go-Money and No-go trials, the fixation cross was preceded by a feedback screen for $750 \mathrm{~ms}$. A successful response provided immediate one dollar reward and feedback, and a response that was either too slow (> $400 \mathrm{~ms}$ ) or too fast ( $<100 \mathrm{~ms}$ ) earned no reward although feedback was provided. The images were alluring because of the immediate one dollar reward. The fourth image was a castle with lights off, and therefore, response was to be withheld. Successful inhibition to the castle yielded a tick and a 10-dollar reward (or no reward for a church). No feedback was provided on monetary amount for delayed reward until the end of the session. Failed inhibition of castle or church resulted in a cross to signify the response was incorrect and immediate reward of one dollar.

\section{Data Processing and Analysis Go/No-go Data}

Behavioral data was assessed using E-Prime 2.0 software (Psychology Software Tools, Pittsburgh, PA, USA), IBM $^{\circledR}$ SPSS $^{\circledR}$ Statistics Version 18 (Chicago, IL, USA), and Microsoft Excel (2007). To assess whether attention diversion improved response inhibition, and whether the outcome of the inhibition influenced performance, a two-way repeated measures analysis of variance (with pair-wise comparisons for significant effects) was conducted. The $F$-statistic was used to determine whether there was a significant difference between means. Confidence intervals were calculated using SPSS and provided additional information regarding the statistical power of each comparison. The dependent variable, mean response inhibition accuracy, was varied by two factors (a) level of attention diversion (High/Low), and (b) future reward (Delayed reward/No delayed reward). Additional secondary analyses were conducted to check for use of cognitive appraisal strategies during response inhibition (e.g., differences in response times between conditions and ensure a minimum level of responding).

\section{Functional Magnetic Resonance Imaging Data}

Functional magnetic resonance imaging data were processed using Analysis of Functional NeuroImages (AFNI) software (Cox, 1996). Following image reconstruction and concatenation of runs, functional data were time-shifted using Fourier interpolation to adjust for difference in slice acquisition times, aligned to corresponding anatomical data, and warped to standard Talairach space. Motion was corrected using threedimensional volume registration with the third volume from the first run as a base. Volumes were blurred using a $4.1 \mathrm{~mm}$ fullwidth half max filter, each voxel was then scaled to a mean of 100 and values over 200 were clipped. To examine the influence of reward and attention diversion on inhibition performance, an event-related analysis was performed that estimated BOLD activity during correct No-go trials. Hemodynamic response functions were calculated using deconvolution of each successful No-go trial response. Activity related to errors, Go-Money trials, feedback screens and motion, were modeled as additional regressors to avoid contamination of baseline and event-related data. TR pairs were censored when the Euclidian Norm of the motion derivative exceeded 1.0. The baseline estimate was the mean activation recorded during the ongoing trial period (Go trials). Thus, the activation observed during successful Nogo trial and Go-Money responses represented activation that differed from that required for the ongoing trial period (or Go) responses. The absence of collinearity between regressors within AFNI X-matrices was confirmed during deconvolution using xmat_tool.py. Event-related map voxels for each regressor of interest were extracted, resampled to anatomical data resolution $\left(1 \mathrm{~mm}^{3}\right)$, and masked using a group-averaged EPI mask dataset. Group activation maps for successful response inhibition were determined with one-sample $t$-tests against the null hypothesis of zero event-related activation changes (i.e., no change related to baseline). Significant voxels passed (a) a voxelwise statistical threshold ( $t=4.84, p \leq 0.0001)$, and (b) a continuity threshold part of a larger $112 \mu \mathrm{l}$ cluster of contiguous significant voxels. The combination of probability and cluster thresholding maximized the power of the statistical test and minimized the likelihood of false positives. Simulation using the 3D ClustSim function (run 
within the whole brain) in AFNI and an uncorrected voxelwise threshold $p=0.0001$, indicated a minimum cluster size of $112 \mu \mathrm{l}$. Activation clusters derived from this whole brain analysis of response inhibition were used to construct activation maps. Whole brain analysis revealed regions of event-related activation during successful No-Go trials. The mean activation for clusters in this map was calculated for the purposes of a functionally derived region of interest (ROI) analysis. ROIs were functionally defined by the No-go $>$ Go contrast. Activation estimates between conditions were compared using two-way repeatedmeasures ANOVA, post hoc pairwise comparisons tested the effects of our experimental manipulations, and corrected using a modified Bonferroni procedure for multiple comparisons (Keppel, 1991).

\section{RESULTS}

\section{Behavioral Data}

The effect of attention diversion on mean response inhibition accuracy in delayed reward and no delayed reward trials of the Go/No-go is shown in Figure 3. There was a significant interaction effect between reward and attention diversion condition on inhibition accuracy, $F(1,20)=6.14, p=0.022$, $\eta_{\mathrm{p}}^{2}=0.24$. For delayed reward trials, mean inhibition accuracy was significantly higher in the high attention diversion condition,

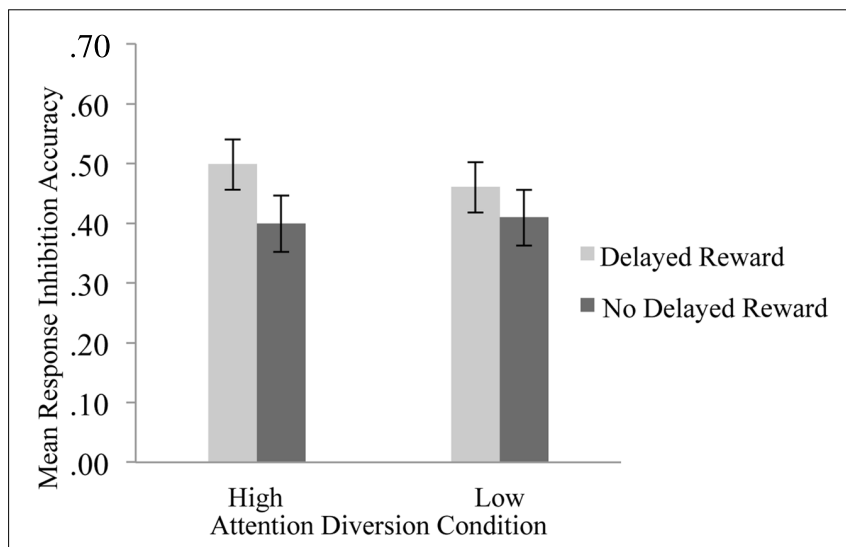

FIGURE 3 | Mean response inhibition accuracy by strength of attention diversion and delayed reward conditions. Error bars represent standard error of the mean. $n=21$. Attention diversion assisted response inhibition performance, but only when inhibition led to delayed gratification.

TABLE 1 | Mean response times in milliseconds by stimuli and attention diversion.

\begin{tabular}{lccc}
\hline Condition & Mean & SD & 95\% CI \\
\hline Go-Money (Churches and Castles)/Face & 381.7 & 55.1 & {$[356.7,406.8]$} \\
Go-Money (Churches and Castles)/ & 388.5 & 54.5 & {$[363.7,413.3]$} \\
Scrambled Face & & & \\
Go (Houses)/Face & 373.7 & 41.2 & {$[354.9,392.5]$} \\
Go (Houses)/Scrambled Face & 385.2 & 42.3 & {$[366.0,404.5]$} \\
\hline
\end{tabular}

$n=21 . S D$, standard deviation; $C l$, confidence interval.
$M=050, S D=0.19,95 \%$ CI $[0.41,0.58]$, than in the low attention diversion condition, $M=0.46, S D=0.20$, 95\% CI $[0.37,0.55]$, but not significantly different for no delayed reward trials, high attention diversion condition, $M=0.40, S D=0.21$, 95\% CI [0.30, 0.50], and low attention diversion condition, $M=0.41, S D=0.22,95 \%$ CI $[0.31,0.51]$. Main effects of delayed reward, $F(1,20)=7.44, p=0.013, \eta_{\mathrm{p}}^{2}=0.27$, and strength of attention diversion, $F(1,20)=0.72, p=0.406$, $\eta_{\mathrm{p}}^{2}=0.04$, were qualified by the significant interaction effect. In summary, attention diversion assisted response inhibition performance, but only when response inhibition led to future reward.

We also examined whether response inhibition improvements were in part due to trade-offs in Go and Go-Money trial response times (RTs). For alluring Go-Money and non-alluring Go trials (houses), overall mean RTs were faster for high attention diversion (face) and non-alluring stimuli (house) conditions (Table 1). There was no significant interaction effect between reward and attention diversion condition on $\mathrm{RTs}, F(1,20)=2.22$, $p=0.152, \eta_{\mathrm{p}}^{2}=0.10$, and no main effect of strength of attention diversion, $F(1,20)=0.96, p=0.338, \eta_{\mathrm{p}}^{2}=0.05$. The main effect of reward condition was significant, $F(1,20)=10.15$, $p=0.005, \eta_{\mathrm{p}}^{2}=0.34$. For low attention diversion trials, RTs were

TABLE 2 | Regions of event-related activation during successful No-go trials.

\begin{tabular}{|c|c|c|c|c|c|}
\hline \multirow[b]{2}{*}{ Structure } & \multirow[b]{2}{*}{ HS $^{\mathbf{a}}$} & \multirow[b]{2}{*}{ Volume $(\mu l)^{b}$} & \multicolumn{3}{|c|}{ Center of Mass ${ }^{c}$} \\
\hline & & & $x$ & $y$ & $z$ \\
\hline Insula & $R^{d}$ & 1888 & 32 & 17 & 12 \\
\hline Insula & $L^{e}$ & 2052 & -29 & 16 & 14 \\
\hline Superior Frontal Gyrus & $\mathrm{R}$ & 1652 & 13 & 48 & 44 \\
\hline Medial Frontal Gyrus* & $\mathrm{R}$ & 999 & 2 & 4 & 54 \\
\hline Medial Frontal Gyrus & $\mathrm{L}$ & 906 & -2 & 46 & 46 \\
\hline Postcentral Gyrus* & $\mathrm{L}$ & 864 & -49 & -19 & 23 \\
\hline Middle Frontal Gyrus & $\mathrm{R}$ & 133 & 36 & 5 & 58 \\
\hline Middle Frontal Gyrus & L & 600 & -36 & 4 & 59 \\
\hline Inferior Frontal Gyrus** & $\mathrm{R}$ & 352 & 41 & -5 & 38 \\
\hline Inferior Frontal Gyrus* & $\mathrm{L}$ & 550 & -41 & 0 & 39 \\
\hline Caudate* & $\mathrm{L}$ & 524 & -14 & 5 & 5 \\
\hline Fusiform Gyrus & $\mathrm{R}$ & 615 & 25 & -59 & -8 \\
\hline Fusiform Gyrus & $\mathrm{L}$ & 279 & -25 & -70 & -6 \\
\hline Inferior Parietal Lobule & $\mathrm{R}$ & 284 & 44 & -70 & 44 \\
\hline Posterior Cingulate & $\mathrm{R}$ & 261 & 1 & -56 & 28 \\
\hline Culmen & $\mathrm{R}$ & 188 & 12 & -69 & -2 \\
\hline Cingulate Gyrus* & $\mathrm{L}$ & 182 & 0 & -44 & 42 \\
\hline Superior Temporal Gyrus* & $\mathrm{R}$ & 149 & 59 & -37 & 10 \\
\hline Superior Temporal Gyrus** & $\mathrm{L}$ & 160 & -61 & -25 & 2 \\
\hline Parahippocampal Gyrus & $\mathrm{R}$ & 113 & 18 & -42 & 8 \\
\hline
\end{tabular}

$n=21$. ${ }^{\mathrm{a}} H S$, hemisphere; ${ }^{\mathrm{b}} \mu L$, microliters. ${ }^{\mathrm{c}}$ Montreal Neurological Institute Brain Atlas Anatomical Coordinates. ${ }^{\mathrm{d}} R$, right; ${ }^{\mathrm{e}} L$, left. ${ }^{*} p<0.05 ;{ }^{* *} p<0.01$. Sidak corrected. The following structures had significant main effects for distractor conditions: left inferior frontal gyrus, caudate, and right and left superior temporal gyri. The following structures had significant main effects for delayed-reward conditions: right inferior frontal gyrus, right medial frontal gyrus, left postcentral gyrus, and left cingulate gyrus. 
significantly faster for Go trials than for Go-Money trials, mean difference $=6.79 \mathrm{~ms}, p=0.013,95 \%$ CI $[1.60,11.99]$, and for high attention diversion trials, RTs were also significantly faster for Go trials than for Go-Money trials, mean difference $=11.55 \mathrm{~ms}$, $p=0.008,95 \%$ CI $[3.35,19.76]$. In summary, mean RTs were faster for Go trials than Go-Money trials. In addition, this difference did not vary as a function of attention diversion strength suggesting accuracy data was representative of response inhibition performance.

\section{Functional Magnetic Resonance Imaging BOLD Activity}

Whole brain analysis revealed 20 regions of event-related activation during successful No-go trials (as shown in Table 2). Event-related ROI analysis was, however, restricted to a priori neural areas (STG, rIFG, and striatum) to avoid reverse inferences (as recommended by Kriegeskorte et al., 2009). Previous literature, including our recent work, has shown these nodes to be implicated in shifts of attention during response inhibition (STG; O'Connor et al., 2012; Lim et al., 2013), goaldirected stopping (rIFG; Aron, 2011), and dorsal striatum in reward-related processes for goal-directed behavior (O'Connor et al., 2012). Therefore, subsequent ROI analyses focussed on the activity of these selected clusters, which were identified as functionally relevant. Also, minimizing the number of ROIs reduced the probability of a type I error (Poldrack, 2007).

Activation maps and percentage BOLD signal change for the STG, left caudate, and rIFG are shown in Figures 4 and 5, and statistical analysis is summarized in Table 3. There was no significant interaction effect between reward and attention diversion condition on any of rSTG, $p=0.135$, left STG (ISTG), $p=0.121$, left caudate, $p=0.451$, or rIFG activation $p=0.428$. Although the interaction effects were non-significant, the behavioral data revealed a context specific effect of our attentional manipulation such that high attention diversion only facilitated inhibitory control when paired with the possibility of delayed reward. In order to assess the neural bases of this effect, pairwise comparisons were conducted. The main effect of delayed reward condition was not significant for rSTG activation, $p=0.134$, ISTG, $p=0.121$, nor left caudate, $p=0.383$, but was significant for rIFG activation, $p=0.001$. The main effect of attention diversion strength on rIFG activation was not significant, $p=0.377$, but was significant for rSTG activation, $p=0.029$, ISTG, $p<0.001$, and left caudate, $p=0.030$. For the rSTG, for delayed reward trials activation was not significantly different between the high and low attention diversion conditions, $p=0.453$, but activation was significantly
A

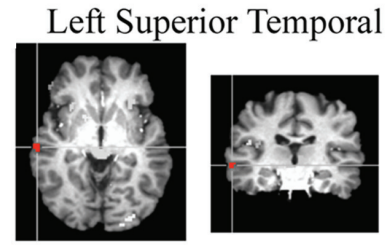

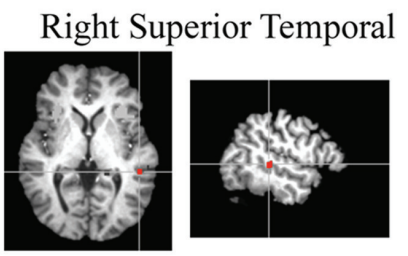

B

BOLD Signal Change for Superior Temporal Gyri by Attention Diversion and Delayed-Reward Conditions

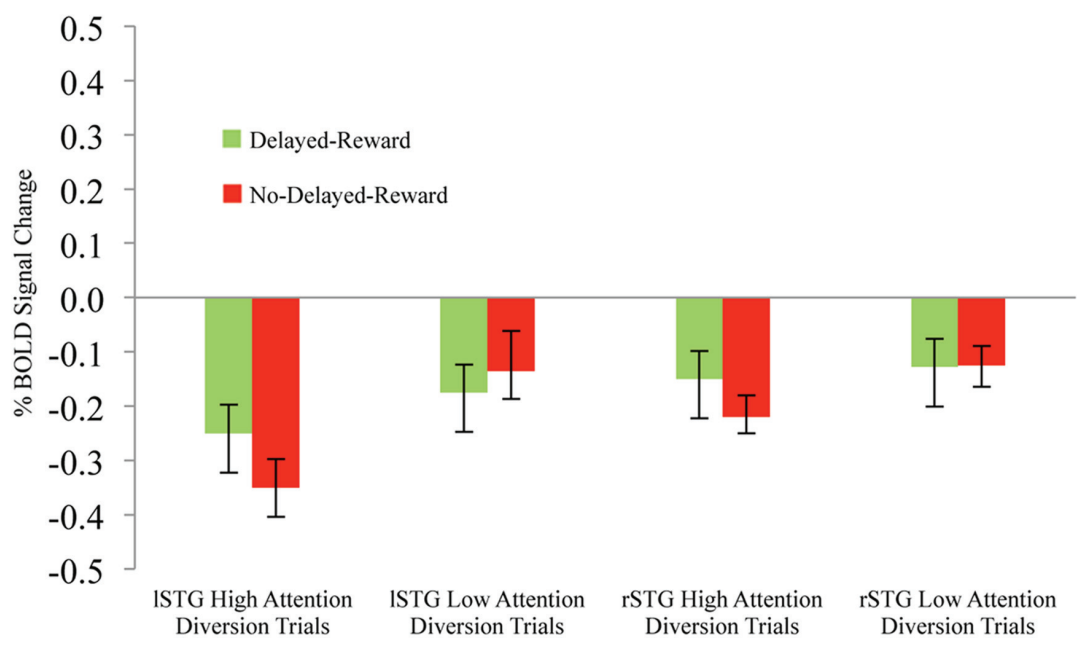

FIGURE 4 | BOLD signal change in superior temporal gyri during successful response inhibition. (A) Activation maps for left and right superior temporal gyri showing significant activity for successful response inhibition, overlaid on coronal brain sections (Talairach template; using AFNI software), $p \leq 0.0001$. (B) Change in BOLD activity plotted by attention diversion and delayed reward conditions. Error bars represent the standard error of the mean. $n=21$. 
A

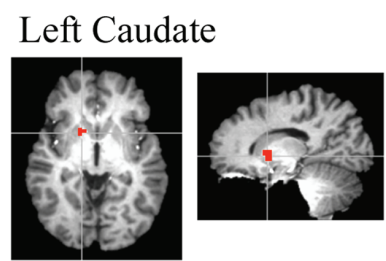

\section{Right Inferior Fontal Gyrus}

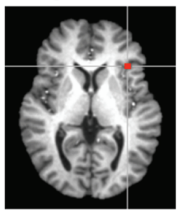

B BOLD Signal Change for left Caudate and right Inferior Frontal Gyrus by Attention Diversion and Delayed-Reward Conditions

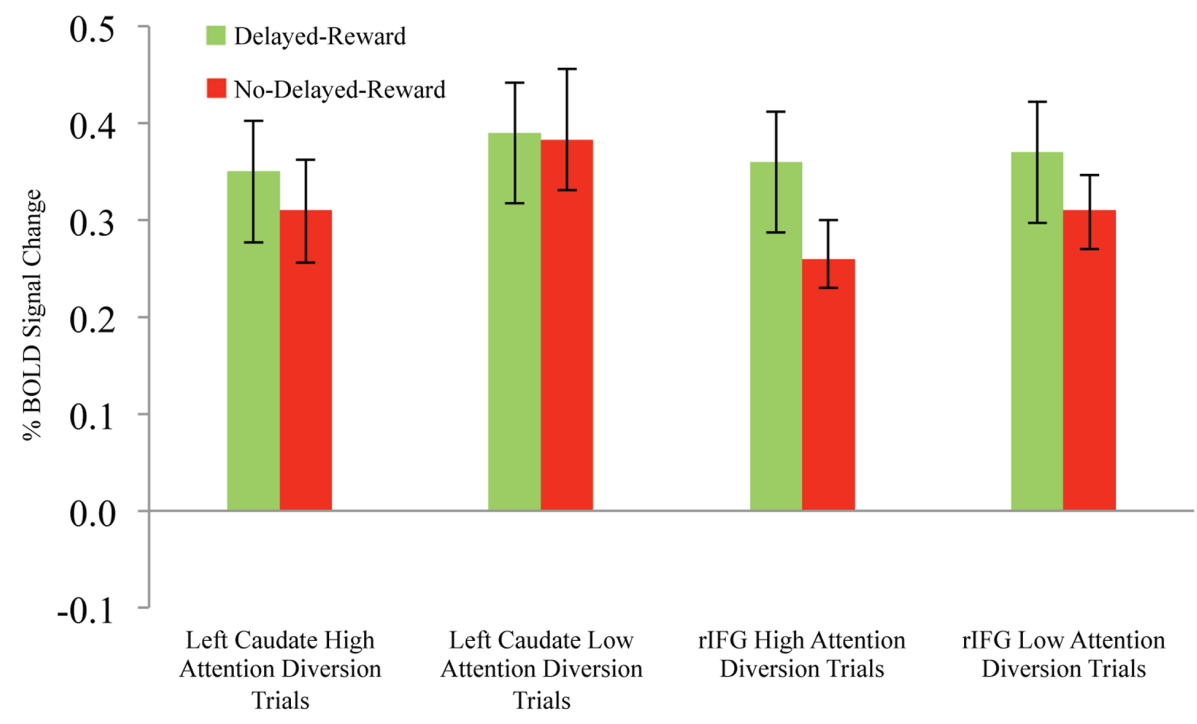

FIGURE 5 | BOLD signal change in left caudate and right inferior frontal gyrus during successful response inhibition. (A) Activation maps for left caudate and right inferior frontal gyrus showing significant activity for successful response inhibition, overlaid on coronal brain sections (Talairach template; using AFNI software), $p \leq 0.0001$. (B) Change in BOLD activity plotted by attention diversion and delayed reward conditions. Error bars represent the standard error of the mean. $n=21$.

lower in the high attention diversion condition than the low attention diversion condition for no delayed reward trials, $p=0.022$. For the ISTG, for no-delayed-reward trials, activation was significantly lower in the high than low attention diversion condition, $p=0.004$, and for delayed reward trials, activation was also significantly lower in the high attention diversion condition than the low attention diversion condition, $p=0.012$. For the left caudate, activation was not significantly different between attention diversion conditions for delayed reward trials, $p=0.229$. For no delayed reward trials, left caudate activation was significantly lower in the high attention diversion condition than the low attention diversion condition, $p=0.029$.

In summary, deactivation of the rSTG was significantly greater for high than low attention diversion condition, but only for no delayed reward trials, and deactivation of the ISTG was significantly greater for the high than low attention diversion condition, for all successful No-go trials. Activation of the left caudate was significantly lower for high than low attention diversion condition, but only for no delayed reward trials, and activation of the rIFG was significantly greater for delayed reward trials than no delayed reward trials, but only for the high attention diversion condition.

\section{DISCUSSION}

In this study, we used exogenous diversions of attention to vary the degree to which participants directed their attention away from alluring stimuli. We found that attention diversion improved response inhibition performance. This finding adds to previous research by providing evidence that response inhibition can be improved by exogenously modulating attention. We also manipulated future benefit in that successful withholding either led to a larger, delayed reward or no delayed reward. Interestingly, we found that attention diversion was only helpful to response inhibition performance, when successful control of impulses ultimately led to a larger, delayed reward. Lack of perceived benefit may, therefore, compromise the application of implicit self-control behaviors.

Regions previously implicated in response inhibition and attention showed sensitivity to context-specific manipulations of attention. Specifically, we observed increased recruitment of the posterior rIFG and enhanced response inhibition performance for delayed reward trials compared to no delayed reward trials. Engagement of posterior rIFG has been associated with selective, goal-directed stopping (Chikazoe et al., 2009; Verbruggen et al., 
TABLE 3 | Region of interest analysis statistical summary.

\begin{tabular}{|c|c|c|c|c|}
\hline Region & Test stastic $F(1,20)$ & $p$-value ${ }^{a}$ & Partial eta squared & Mean difference $[95 \% \mathrm{Cl}]$ \\
\hline \multicolumn{5}{|l|}{ Left Superior Temporal Gyrus, coordinates ${ }^{b}$} \\
\hline \multicolumn{5}{|l|}{$x$ y $z,-61-252$, volume $160 \mu \mathrm{l}$} \\
\hline Interaction effect & 0.58 & 0.121 & 0.12 & \\
\hline Main effect for delayed reward & 2.63 & 0.121 & 0.12 & \\
\hline Main effect for strength of attention diversion (AD) & 27.14 & $<0.001$ & 0.58 & \\
\hline No delayed reward condition (High less Low AD strength) & & 0.004 & & $-0.18[-0.29,-0.06]$ \\
\hline Delayed reward condition (High less Low AD strength) & & 0.012 & & $-0.12[-0.21,-0.03]$ \\
\hline \multicolumn{5}{|l|}{$\begin{array}{l}\text { Right Superior Temporal Gyrus, coordinates }{ }^{b} \\
x y z, 59-3710, \text { volume } 149 \mu \text { I }\end{array}$} \\
\hline Interaction effect & 2.42 & 0.135 & 0.11 & \\
\hline Main effect for delayed reward & 2.44 & 0.134 & 0.11 & \\
\hline Main effect for AD strength & 5.55 & 0.029 & 0.22 & \\
\hline No delayed reward condition (High less Low AD strength) & & 0.022 & & $-0.95[-0.17,-0.02]$ \\
\hline Delayed reward condition (High less Low AD strength) & & 0.453 & & $-0.02[-0.08,0.04]$ \\
\hline \multicolumn{5}{|l|}{ Left Caudate, coordinates $^{b}$ x y z,-14 5 5, volume $524 \mu$ l } \\
\hline Interaction effect & 0.59 & 0.451 & 0.03 & \\
\hline Main effect for delayed reward & 0.80 & 0.383 & 0.04 & \\
\hline Main effect for $A D$ strength & 5.43 & 0.030 & 0.21 & \\
\hline No delayed reward condition (High less Low AD strength) & & 0.029 & & $-0.07[-0.14,-0.01]$ \\
\hline Delayed reward condition (High less Low AD strength) & & 0.229 & & $-0.04[-0.11,0.03]$ \\
\hline \multicolumn{5}{|l|}{$\begin{array}{l}\text { Right Inferior Frontal Gyrus, coordinates } \\
x y z, 59-3710, \text { volume } 352 \mu \text { I }\end{array}$} \\
\hline Interaction effect & 0.66 & 0.428 & 0.03 & \\
\hline Main effect for delayed reward & 0.82 & 0.377 & 0.04 & \\
\hline Main effect for AD strength & 14.21 & 0.001 & 0.42 & \\
\hline No delayed reward condition (High less Low AD strength) & & 0.080 & & $0.06[-0.01,0.13]$ \\
\hline Delayed reward condition (High less Low AD strength) & & 0.003 & & $0.09[0.04,0.15]$ \\
\hline
\end{tabular}

$n=21$. Cl, confidence interval. a Sidak corrected; b Peak Montreal Neurological Institute Brain Atlas Anatomical Coordinates.

2010; Aron, 2011). rIFG activation did not vary across the attention diversion conditions. However, STG were more deactivated for high attention diversion trials than low. Increased deactivation of STG is interpreted to be indicative of a degree of disengagement from salient (immediately rewarding) stimuli, facilitating successful response inhibition (O'Connor et al., 2012).

Shifts of attention away from target stimuli might also be reflected in reduced activity in reward-related neural processes. Our finding of increased deactivation of the left caudate for higher attention diversion trials was consistent with this concept. Dorsal striatal activity has been associated with elevated target saliency (Pizzagalli et al., 2009; Onoda et al., 2011), and computation of relative value between outcomes or in comparison to expected reward (O’Doherty et al., 2004; Balleine and O'Doherty, 2010; Wunderlich et al., 2012). The inference is that the encoding of value potentially informs prefrontal executive processes, consistent with a system that computes value and best action-outcome (Frydman et al., 2011). However, our left caudate results were only significant for the no delayed reward condition and not the delayed reward condition. Other regions, such as the ventral striatum and orbitofrontal cortex, have been consistently linked to reward-related processes (Fitzgerald et al., 2009; Plassmann et al., 2010). Comparison of BOLD activity between reward and attention diversion conditions did not detect any significant change in these regions during successful response inhibition. However, the context for reward in our study is different, which may explain why the conditions do not vary.

Our findings may have relevance in a number of health issues where impaired self-control is thought to be factor. Some clinical populations are known to exhibit compromised response inhibition and high reward sensitivity (e.g., addiction, Goldstein and Volkow, 2002; Dackis and O'Brien, 2005). Deficits in using shifts of attention to diffuse target saliency may be critical for the initiation and continuation of self-regulatory lapses (e.g., rumination in residual depression and relapse associated with exposure to conditioned drug-cues in addiction). It would be instructive to understand whether differences in these selfregulatory processes between individuals vary over time (Kane and Engle, 2002; Unsworth and Engle, 2007; Friedman et al., 2008; Casey et al., 2011), are reflected in neural circuitry or functioning of the dopaminergic network (Buckholtz et al., 2010), and amenable to training (Klingberg, 2010). Our findings indicate that perceived lack of future benefit may undermine the application of such implicit self-control behaviors. In public health policy, measures to diffuse target saliency and emphasize future benefit may aid resistance to immediate reward. For instance, plain paper packaging of cigarettes may help reduce 
cigarette saliency, and extolling the future health benefits of stopping smoking may provide a more tangible delayed reward for smokers. Also, a perception that successful resistance to an immediate reward brings uncertain future benefit may somewhat deplete response inhibition capacity, resulting in more impulsive behavior (Milkman, 2012).

The experimental design was a practical way to mimic realworld situations as the MRI scanner environment is highly constrained. However, a caveat is that our laboratory findings may not translate to everyday circumstances. Delay to reward was constrained by the duration of the laboratory session and might not be analogous to real-life delayed reward. The facilitating effect of faces on response inhibition may not generalize to other situations. While it is difficult to determine the authenticity of participants' efforts to adhere to inhibition instructions or their willingness to engage properly in the exercise, analysis of performance markers (e.g., response times, accuracy, nonresponse to Go trials) indicated a high level of engagement and diligence. These results indicate that improved response inhibition accuracy on high attention diversion/delayed reward No-go trials cannot be attributed to slower response speed. In addition, people responded more quickly to Go-trials (houses) than Go-Money trials (churches and castles), which was expected given that identification of a single feature (building type for house) was sufficient to respond to the house, compared to two features for churches and castles (building type and lights). Also, high levels of non-response to Go-Money trials would have implied a strategy to maximize No-go gain, and similarly, relatively low accuracy (less than 10\%) for No-go trials may have indicated difficulty with the task or a strategy of maximizing immediate rewards. There was, however, no evidence of strategies to maximize immediate or delayed reward. Moreover, block order was counterbalanced throughout the experiment to mitigate possible learning effects, and we used inter-block "Go task" runs to mitigate effects of reversal learning on subsequent blocks and reinforce immediate reward association irrespective of previous contingencies.

Future research could extend the present study by utilizing gradational changes to attention diversion (face) stimuli in order to expand understanding of the functional relationship between

\section{REFERENCES}

Armel, K. C., Beaumel, A., and Rangel, A. (2008). Biasing simple choices by manipulating relative visual attention. Judgm. Decis. Mak. 3, 396-403.

Aron, A. R. (2011). From reactive to proactive and selective control: developing a richer model for stopping inappropriate responses. Biol. Psychiat. 69, e55-e68. doi: 10.1016/j.biopsych.2010.07.024

Balleine, B. W., Delgado, M. R., and Hikosaka, O. (2007). The role of the dorsal striatum in reward and decision-making. J. Neurosci. 27, 8161-8165. doi: 10.1523/JNEUROSCI.1554-07.2007

Balleine, B. W., and O'Doherty, J. P. (2010). Human and rodent homologies in action control: corticostriatal determinants of goal-directed and habitual action. Neuropsychopharmacology 35, 48-69. doi: 10.1038/npp.2009.131

Baumeister, R. F., and Heatherton, T. F. (1996). Self-regulation failure: an overview. Psychol. Inq. 7, 1-15. doi: 10.1207/s15327965pli0701_1

Boonstra, A. M., Kooij, J. J. S., Oosterlaan, J., Sergeant, J. A., and Buitelaar, J. K. (2010). To act or not to act, that's the problem: primarily inhibition difficulties in adult ADHD. Neuropsychology 24, 209-221. doi: 10.1037/a0017670 attention diversion, reward, and response inhibition. The methodology has been used successful in recent research (e.g., Preuschoff et al., 2008), but the high number of events necessary for sufficient power precluded integration of the methodology within the current study. In addition, a modified task comprising of explicitly stated temporal delays with real waiting periods before rewarding successful inhibition performance would lend greater credibility to the suggestion that this type of task represents a fusion of response inhibition (impulsive action) and delay discounting (impulsive choice).

This study adds to previous literature of the importance of an attentional mechanism for successful response inhibition of alluring stimuli. In the present experimental paradigm, inhibition appears to be a set of distinct neural processes related to stopping and the ability to implicitly control attention, which might be a target for intervention (training). This study also highlighted the importance of perceived future benefit for these implicit self-regulatory behaviors.

\section{AUTHOR CONTRIBUTIONS}

DO and FS designed the study, and collected, analyzed , and interpreted the data. FS drafted the manuscript, and DO and $\mathrm{RH}$ revised the manuscript. $\mathrm{RH}, \mathrm{CO}$, and $\mathrm{KM}$ contributed to study design. All authors discussed the results and commented on the manuscript.

\section{FUNDING}

The research was supported by the Australian Research Council DP1092852.

\section{ACKNOWLEDGMENTS}

The authors gratefully acknowledge contributions to data collection by Sarah Rossiter and Kathleen Charles-Walsh, and neuroimage data acquisition by Richard Nibbs.

Brody, A. L., Mandelkern, M. A., Olmstead, R. E., Jou, J., Tiongson, E., Allen, V., et al. (2007). Neural substrates of resisting craving during cigarette cue exposure. Biol. Psychiatry 62, 642-651. doi: 10.1016/j.biopsych.2006. 10.026

Buckholtz, J. W., Treadway, M. T., Cowan, R. L., Woodward, N. D., Li, R., Ansari, M. S., et al. (2010). Dopaminergic network differences in human impulsivity. Science 329:532. doi: 10.1126/science.1185778

Casey, B. J., Somerville, L. H., Gotlib, I. H., Ayduk, O., Franklin, N. T., Askren, M. K., et al. (2011). Behavioral and neural correlates of delay of gratification 40 years later. Proc. Natl. Acad. Sci. U.S.A. 108, 14998-15003. doi: 10.1073/pnas. 1108561108

Chikazoe, J., Jimura, K., Asari, T., Yamashita, K., Morimoto, H., Hirose, S., et al. (2009). Functional dissociation in right inferior frontal cortex during performance of Go/No-Go task. Cereb. Cortex 19, 146-152. doi: 10.1093/cercor/bhn065

Cox, R. W. (1996). AFNI: software for analysis and visualization of functional magnetic resonance neuroimages. Comput. Biomed. Res. 29, 162-173. doi: 10.1006/cbmr.1996.0014 
Dackis, C., and O’Brien, C. (2005). Neurobiology of addiction: treatment and public policy ramifications. Nat. Neurosci. 8, 1431-1436. doi: 10.1038/nn11051431

Farah, M. J. (1996). Is face recognition 'special'? Evidence from neuropsychology. Behav. Brain Res. 76, 181-189. doi: 10.1016/0166-4328(95)00198-0

Fillmore, M. T., and Rush, C. R. (2002). Impaired inhibitory control of behavior in chronic cocaine users. Drug Alcohol Depend. 66, 265-273. doi: 10.1016/s03768716(01)00206-x

Fitzgerald, T. H., Seymour, B., and Dolan, R. J. (2009). The role of human orbitofrontal cortex in value comparison for incommensurable objects. J. Neurosci. 29, 8388-8395. doi: 10.1523/jneurosci.0717-09.2009

Friedman, N. P., Miyake, A., Young, S. E., De Fries, J. C., Corley, R. P., and Hewitt, J. K. (2008). Individual differences in executive functions are almost entirely genetic in origin. J. Exp. Psychol. 137, 201-225. doi: 10.1037/00963445.137.2.201

Frydman, C., Barberis, N., Camerer, C., Bossaerts, P., and Rangel, A. (2011). Testing Theories of Investor Behavior Using Neural Data. Working Paper: Caltech. Available at: http://ssrn.com/abstract $=1892338$. doi: $10.2139 /$ ssrn. 18 92338

Goldstein, R. Z., and Volkow, N. D. (2002). Drug addiction and its underlying neurobiological basis: neuroimaging evidence for the involvement of the frontal cortex. Am. J. Psychiat. 159, 1642-1652. doi: 10.1176/appi.ajp.159. 10.1642

Goudriaan, A. E., Oosterlaan, J., De Beurs, E., and van den Brink, W. (2006). Neurocognitive functions in pathological gambling: a comparison with alcohol dependence. Tourette syndrome and normal controls. Addiction 101, 534-547. doi: $10.1111 / \mathrm{j} .1360-0443.2006 .01380 . \mathrm{x}$

Hampshire, A., and Sharp, D. J. (2015). Contrasting network and modular perspectives on inhibitory control. Trends Cogn. Sci. 19, 445-452. doi: 10.1016/j.tics.2015.06.006

Hampton, A. N., Bossaerts, P., and O’Doherty, J. P. (2008). Neural correlates of mentalizing-related computations during strategic interactions in humans. Proc. Natl. Acad. Sci. U.S.A. 105, 6741-6746. doi: 10.1073/pnas.0711099105

Hare, T. A., Camerer, C. F., Knoepfle, D. T., O’Doherty, J. P., and Rangel, A. (2010). Value computations in ventral medial prefrontal cortex during charitable decision making incorporate input from regions involved in social cognition. J. Neurosci. 30, 583-590. doi: 10.1523/jneurosci.4089-09.2010

Heatherton, T. F., and Wagner, D. D. (2011). Cognitive neuroscience of selfregulation failure. Trends Cogn. Sci. 15, 132-139. doi: 10.1016/j.tics.2010.12.005

Kane, M. J., and Engle, R. W. (2002). The role of prefrontal cortex in working-memory capacity, executive attention, and general fluid intelligence: an individual-differences perspective. Psychon. B. Rev. 9, 637-671. doi: 10.3758/bf03196323

Keppel, G. (1991). Design and Analysis: A Researcher's Handbook. Upper Saddle River, NJ: Prentice Hall.

Klingberg, T. (2010). Training and plasticity of working memory. Trends Cogn. Sci. 14, 317-324. doi: 10.1016/j.tics.2010.05.002

Kriegeskorte, N., Simmons, W. K., Bellgowan, P. S. F., and Baker, C. I. (2009). Circular analysis in systems neuroscience: the dangers of double dipping. Nat. Neurosci. 12, 535-540. doi: 10.1038/nn.2303

Lavie, N., Ro, T., and Russell, C. (2003). The role of perceptual load in processing distractor faces. Psychol. Sci. 14, 510-515. doi: 10.1111/1467-9280. 03453

Li, C. R., and Sinha, R. (2008). Inhibitory control and emotional stress regulation: neuroimaging evidence for frontal-limbic dysfunction in psycho-stimulant addiction. Neurosci. Biobehav. R. 32, 581-597. doi: 10.1016/j.neubiorev.2007.10.003

Lim, S. L., O'Doherty, J. P., and Rangel, A. (2011). The decision value computations in the vmPFC and striatum use a relative value code that is guided by visual attention. J. Neurosci. 31, 13214-13223. doi: 10.1523/JNEUROSCI.124611.2011

Lim, S. L., O’Doherty, J. P., and Rangel, A. (2013). Stimulus value signals in ventromedial PFC reflect the integration of attribute value signals computed in fusiform gyrus and posterior superior temporal gyrus. J. Neurosci. 33, 8729-8741. doi: 10.1523/jneurosci.4809-12.2013

Macknik, S. L., and Livingstone, M. S. (1998). Neuronal correlates of visibility and invisibility in the primate visual system. Nat. Neurosci. 1, 144-149. doi: $10.1038 / 393$
Milkman, K. L. (2012). Unsure what the future will bring? You may overindulge: uncertainty increases the appeal of wants over shoulds. Organ. Behav. Hum. Dec. 119, 163-176. doi: 10.1016/j.obhdp.2012.07.003

Mischel, W., and Baker, N. (1975). Cognitive appraisals and transformations in delay behavior. J. Pers. Soc. Psychol. 31, 254-261. doi: 10.1037/h0076272

Mischel, W., and Ebbesen, E. B. (1970). Attention in delay of gratification. J. Pers. Soc. Psychol. 16, 329-337. doi: 10.1037/h0029815

Mischel, W., Ebbesen, E. B., and Raskoff Zeiss, A. (1972). Cognitive and attentional mechanisms in delay of gratification. J. Pers. Soc. Psychol. 21, 204-218. doi: $10.1037 / \mathrm{h} 0032198$

Mischel, W., Shoda, Y., and Peake, P. K. (1988). The nature of adolescent competencies predicted by preschool delay of gratification. J. Pers. Soc. Psychol. 54, 687-696. doi: 10.1037/0022-3514.54.4.687

Monterosso, J. R., Aron, A. R., Cordova, X., Xu, J., and London, E. D. (2005). Deficits in response inhibition associated with chronic methamphetamine abuse. Drug Alcohol Depend. 79, 273-277. doi: 10.1016/j.drugalcdep.2005.02.002

Ochsner, K. N., and Gross, J. J. (2005). The cognitive control of emotion. Trends Cogn. Sci. 9, 242-249. doi: 10.1016/j.tics.2005.03.010

O’Connor, D. A., Rossiter, S., Yücel, M., Lubman, D. I., and Hester, R. (2012). Successful inhibitory control over an immediate reward is associated with attentional disengagement in visual processing areas. NeuroImage 62, 18411847. doi: 10.1016/j.neuroimage.2012.05.040

O’Doherty, J. P., Dayan, P., Schultz, J., Deichmann, R., Friston, K., and Dolan, R. J. (2004). Dissociable roles of ventral and dorsal striatum in instrumental conditioning. Science 304, 452-454. doi: 10.1126/science.1094285

Oldfield, R. C. (1971). The assessment and analysis of handedness: the Edinburgh inventory. Neuropsychologia 9, 97-113. doi: 10.1016/0028-3932(71)90067-4

Onoda, K., Okamoto, Y., Kunisato, Y., Aoyama, S., Shishida, K., Okada, G., et al. (2011). Inter-individual discount factor differences in reward prediction are topographically associated with caudate activation. Exp. Brain Res. 212, 593-601. doi: 10.1007/s00221-011-2771-3

Padmala, S., and Pessoa, L. (2010). Interactions between cognition and motivation during response inhibition. Neuropsychologia 48, 558-565. doi: 10.1016/j.neuropsychologia.2009.10.017

Penadés, R., Catalán, R., Rubia, K., Andrés, S., Salamero, M., and Gastó, C. (2007). Impaired response inhibition in obsessive compulsive disorder. Eur. Psychiat. 22, 404-410. doi: 10.1016/j.eurpsy.2006.05.001

Peters, J., and Büchel, C. (2011). The neural mechanisms of inter-temporal decision-making: understanding variability. Trends Cogn. Sci. 15, 227-239. doi: 10.1016/j.tics.2011.03.002

Pizzagalli, D. A., Holmes, A. J., Dillon, D. G., Goetz, E. L., Birk, J. L., Ryan Bogdan, A. M., et al. (2009). Reduced caudate and nucleus accumbens response to rewards in unmedicated individuals with major depressive disorder. Am. J. Psychiat. 166, 702-710. doi: 10.1176/appi.ajp.2008.08 081201

Plassmann, H., O’Doherty, J., and Rangel, A. (2010). Appetitive and aversive goal values are encoded in the medial orbitofrontal cortex at the time of decision making. J. Neurosci. 30, 10799-10808. doi: 10.1523/jneurosci.0788-10. 2010

Poldrack, R. A. (2007). Region of interest analysis for fMRI. Soc. Cogn. Affect. Neur. 2, 67-70. doi: 10.1093/scan/nsm006

Preuschoff, K., Quartz, S. R., and Bossaerts, P. (2008). Human insula activation reflects risk prediction errors as well as risk. J. Neurosci. 28, 2745-2752. doi: 10.1523/JNEUROSCI.4286-07.2008

Rosen, B. R., Buckner, R. L., and Dale, A. M. (1998). Event-related functional MRI: past, present, and future. Proc. Natl. Acad. Sci. U.S.A. 95, 773-780. doi: 10.1073/pnas.95.3.773

Tabibnia, G., Monterosso, J. R., Baicy, K., Aron, A. R., Poldrack, R. A., Chakrapani, S., et al. (2011). Different forms of self-control share a neurocognitive substrate. J. Neurosci. 31, 4805-4810. doi: 10.1523/jneurosci. 2859-10.2011

Unsworth, N., and Engle, R. W. (2007). The nature of individual differences in working memory capacity: active maintenance in primary memory and controlled search from secondary memory. Psychol. Rev. 114, 104-132. doi: 10.1037/0033-295x.114.1.104

Verbruggen, F., Aron, A. R., Stevens, M. A., and Chambers, C. D. (2010). Theta burst stimulation dissociates attention and action updating in human 
inferior frontal cortex. Proc. Natl. Acad. Sci. U.S.A. 107, 13966-13971. doi: 10.1073/pnas. 1001957107

Volkow, N. D., Fowler, J. S., Wang, G. J., Telang, F., Logan, J., Jayne, M., et al. (2010). Cognitive control of drug craving inhibits brain reward regions in cocaine abusers. NeuroImage 49, 2536-2543. doi: 10.1016/j.neuroimage.2009.10.068

Vuilleumier, P., and Schwartz, S. (2001). Emotional facial expressions capture attention. Neurology 56, 153-158. doi: 10.1212/wnl.56.2.153

Wunderlich, K., Dayan, P., and Dolan, R. J. (2012). Mapping value based planning and extensively trained choice in the human brain. Nat. Neurosci. 15, 786-791. doi: 10.1038/nn.3068
Conflict of Interest Statement: The authors declare that the research was conducted in the absence of any commercial or financial relationships that could be construed as a potential conflict of interest.

Copyright () 2016 Scalzo, O'Connor, Orr, Murphy and Hester. This is an openaccess article distributed under the terms of the Creative Commons Attribution License (CC BY). The use, distribution or reproduction in other forums is permitted, provided the original author(s) or licensor are credited and that the original publication in this journal is cited, in accordance with accepted academic practice. No use, distribution or reproduction is permitted which does not comply with these terms. 